\title{
Systems Approach to Standardization of Care in the Secondary Prevention of Noncardioembolic Ischemic Stroke
}

\author{
Alpesh Amin, MD, MBA ${ }^{1}$ \\ Daniel David Dressler, MD, Msc $^{2}$ \\ David J. Likosky, MD ${ }^{3}$ \\ Kiwon Lee, ${ }^{4}{ }^{4}$ \\ Dara G. Jamieson, MD $^{5}$ \\ David Krakow, MD ${ }^{6}$ \\ Saad Rahman, MD $^{7}$ \\ David M. Brown, MD ${ }^{8}$
}

\footnotetext{
${ }^{1}$ Executive Director, Hospitalist Program, Vice Chair for Clinical Affairs and Quality, Department of Medicine, University of California, Irvine School of Medicine, Orange, California.

${ }^{2}$ Director of Hospital Medicine, Emory University Hospital, Assistant Professor of Internal Medicine, Emory University School of Medicine, Atlanta, Georgia.

${ }^{3}$ Medical Director, Evergreen Neuroscience Institute, Hospitalist/Neurologist, Division of Hospitalist Medicine/Neurology, Evergreen Hospital Medical Center, Kirkland, Washington.

${ }^{4}$ Assistant Professor, Neurology and Neurosurgery, Columbia University College of Physicians and Surgeons, New York, New York.

${ }^{5}$ Associate Professor of Clinical Neurology, Weill Medical College of Cornell University, New York, New York.

${ }^{6}$ Assistant Professor of Medicine, Assistant Director of Hospital Medicine, Emory University Hospital, Atlanta, Georgia.

${ }^{7}$ Swedish Adult Hospitalist, Swedish Medical Center, Seattle, Washington.

${ }^{8}$ Medical Director, Stroke Program, Hoag Memorial Hospital Presbyterian Neuroscience Institute, Newport Beach, California.
}

A systems approach to stroke care is a multidisciplinary team strategy with the goal of reducing stroke risk. This strategy may start with community-based primary prevention education. For individuals who experience stroke, the inpatient component of a stroke system is designed to integrate evidence-based acute treatment and secondary prevention strategies. A key component to stroke systems care is the use of standardized, evidence-based treatment protocols. During the acute (or subacute) phase of care, the team must be prepared to prevent stroke progression, prevent complications of stroke, manage comorbidities, and reduce the risk of secondary stroke. Hospitalists may take a leadership role both in establishing stroke systems protocols at their institutions and in providing inpatient care for stroke patients. The objective of this article is to review the components of inpatient stroke systems, with a focus on care of patients with noncardioembolic ischemic stroke. Methods to minimize common barriers to establishing stroke systems programs are also presented. Journal of Hospital Medicine 2008;3(4 Suppl):S29-S35. @ 2008 Society of Hospital Medicine.

KEYWORDS: hospitalist inpatient management, noncardioembolic stroke, ischemic stroke, stroke systems protocols.

D espite the considerable national attention drawn to the need for improved secondary stroke prevention, a gap remains between evidence and application for stroke and other vascular events. Experience with the Coverdell stroke registry has shown that a minority of acute stroke patients receive the care recommended in established guidelines. ${ }^{1}$ Data collected from 4 registry centers in the United States showed a consistent lack of appropriate diagnostics, patient education, and initiation of drug therapies proven to reduce the risk of recurrent stroke. ${ }^{1}$

According to a report from the Committee on the Quality of Healthcare in America published in 2001, suboptimal treatment as well as inefficient use of health resources can be largely attributed to fragmentation of health care delivery in the management of various diseases in the United States. ${ }^{2}$ In response to these findings, the American Stroke Association (ASA) has established recommendations for the development of stroke systems of care. The objective of a systems approach is to integrate preventive and treatment services and provide patients with evidence-based care. ${ }^{3}$

During hospitalization for acute stroke, immediate treatment must focus on minimizing stroke progression, avoiding common 
complications, and preventing recurrent stroke. Prior to discharge, patients need to be educated about the importance of lifestyle modifications and pharmacotherapies to reduce their risk of a recurrence of the stroke and other atherosclerotic vascular events. ${ }^{3}$ As the physicians who focus on inpatient care, hospitalists are likely to be responsible for participating in and coordinating the multidisciplinary team that provides treatment and services to stroke patients. Hospitalists also must facilitate the transition from inpatient to outpatient care. Hospitalists are in a position to help educate stroke patients about prevention strategies throughout the hospitalization period. These functions provide hospitalists with the opportunity to lead, coordinate, and participate in stroke systems care at their institutions.

The present article discusses the components of stroke systems care recommended by the ASA and the best-practices recommendations from the recent hospitalist roundtable discussion on routine acute stroke care. The national treatment guidelines and clinical trials supporting the recommendations of the hospitalist roundtable participants have been discussed in the article in this supplement by Dr. Likosky et al, as well as in the patient scenarios article in this supplement by Dr. Lee et al. Some of the anticipated barriers and pitfalls that may be encountered, along with potential solutions, are also discussed. Hospitalists may be able to use this review to adapt feasible components of the systems care for stroke management to improve care at their institutions.

\section{WHAT IS STROKE SYSTEMS CARE?}

A stroke system is coordinated stroke care along the entire continuum from primary prevention to rehabilitation. Post-emergency department inpatient care for patients with acute stroke, also referred to as subacute care, is only one component of the community-based stroke systems of care recommended by the ASA (Fig. 1). ${ }^{3}$ In this model, regional stroke systems identify hospitals that are "acute stroke capable" and determine that those institutions use clinical pathways that reflect well-established standards of care and nationally recognized guidelines. ${ }^{3}$ In this broad sense of the term, stroke systems function to organize and coordinate the various agencies and health care providers responsible for caring for patients with stroke, from the first call to emergency services

\section{Prehospital}

Emergency care and transport

Acute care

Emergency department

\section{Hospitalist-based subacute inpatient care \\ Prevent stroke progression Prevent complications Manage comorbidities Prevent secondary stroke}

\section{Postdischarge \\ Outpatient care}

FIGURE 1. Components of stroke systems care of patients with stroke with hospitalist-based inpatient management.

through postdischarge medical care and rehabilitation (Table 1). The subacute phase of care provides the bridge from management of the medical emergency to discharge and is central to secondary stroke prevention.

\section{RATIONALE FOR HOSPITAL-BASED STROKE SYSTEMS}

The Preventing Recurrence of Thrombo-embolic Events through Coordinated Treatment (PROTECT) program provides proof of concept. ${ }^{4}$ The PROTECT program was implemented at a large teaching hospital to improve diagnosis, treatment, and secondary prevention for patients with ischemic stroke. ${ }^{4}$ Four medication goals and instruction in 4 lifestyle interventions were chosen as indicators of program impact. In the first year after PROTECT was started, $100 \%$ of eligible patients received instruction in all 4 areas of lifestyle change prior to discharge. ${ }^{4}$ In the year following 
TABLE 1

Three Critical Functions of a Stroke System as Recommended by the American Stroke Association

1. Ensure effective interaction and collaboration among agencies, services, and people involved in providing prevention and timely identification, transport, treatment, and rehabilitation of individual stroke patients in a locality or region.

2. Promote the use of an organized standardized approach at each facility and component of the system.

3. Identify performance measures (both process and outcomes measures) and include a mechanism for evaluating the effectiveness through which the entire system and its individual components continue to evolve and improve.

Text reprinted with permission from Schwamm LH et al. Circulation. 2005;111:1078-1091. ${ }^{3}$

implementation of PROTECT, the rate of appropriate prescribing of antithrombotics was $98 \%$. Appropriate prescribing of angiotensin-converting enzyme (ACE) inhibitors or angiotensin receptor blockers (ARBs), statins, and thiazide diuretics was significantly increased from pre-PROTECT levels. ${ }^{4}$ After 3 months of follow-up, patient adherence to therapy remained high. ${ }^{5}$ The final results of the PROTECT program are not yet available; however, it is reasonable to expect that increased use of evidence-based therapy and good patient adherence to these proven therapies will have led to better patient outcomes, including lower rates of recurrent stroke.

Patient outcomes data are available for a related initiative for treatment of patients hospitalized with myocardial infarction. Compared with the year prior to implementation of the Cardiac Hospitalization Atherosclerotic Management Program (CHAMP), more patients who were involved in the CHAMP intervention achieved low-density lipoprotein cholesterol levels $<I=100 \mathrm{mg} / \mathrm{dL}(6 \%$ vs $58 \%, P<.001)$. In addition, these patients achieved a $57 \%$ reduction in recurrent myocardial infarction. ${ }^{6}$

These 2 studies indicate a benefit of establishing hospital-based stroke systems; however, these studies are the initial steps, and each has limitations. For example, neither study was a prospective, randomized trial with a concurrent control group. $^{4,6}$ In addition, PROTECT data were not evaluated by independent audit but by individuals who were aware of the program goals, and limited data were available regarding contraindications to therapy. ${ }^{4}$ CHAMP did not assess adherence to nonpharmacologic interventions or the effect of surgical interventions. ${ }^{6}$ Large, randomized, controlled trials are needed to better understand the
TABLE 2

Barriers and Solutions to Implementing In-Hospital Secondary Prevention Strategies

\begin{tabular}{|c|c|}
\hline Barriers & Solutions \\
\hline 1. Lack of proof of concept. & $\begin{array}{l}\text { 1. PROTECT demonstrates improved } \\
\text { stroke care. }\end{array}$ \\
\hline $\begin{array}{l}\text { 2. Lack of ownership: acute versus } \\
\text { chronic disease dilemma. }\end{array}$ & $\begin{array}{l}\text { 2. View hospital as capture point for } \\
\text { patients with chronic diseases. }\end{array}$ \\
\hline 3. Lack of financial incentives. & $\begin{array}{l}\text { 3. JCAHO/NCQA will measure and } \\
\text { report to payers. }\end{array}$ \\
\hline $\begin{array}{l}\text { 4. Communication gaps-neurologists, } \\
\text { hospitalists, and primary care } \\
\text { physicians. }\end{array}$ & $\begin{array}{l}\text { 4. Education and mobilization of case } \\
\text { management teams. }\end{array}$ \\
\hline $\begin{array}{l}\text { 5. Poor standardization of orders and } \\
\text { testing procedures. }\end{array}$ & $\begin{array}{l}\text { 5. Written protocols for diagnosis and } \\
\text { treatment; written orders. }\end{array}$ \\
\hline 6. Lack of tools and resources. & $\begin{array}{l}\text { 6. JCAHO, "Get with the Guidelines," } \\
\text { and PROTECT Web sites. }\end{array}$ \\
\hline
\end{tabular}

JCAHO, Joint Commission on Accreditation of Healthcare Organizations; NCQA, National Committee for Quality Assurance; PROTECT, Preventing Recurrent Thromboembolic Events Through Coordinated Treatment.

impact of such systems. Although larger evidencebased trials are needed, it is important to review available information on stroke systems to adapt those components that align with each institution's available resources.

\section{ESTABLISHING HOSPITAL-BASED STROKE SYSTEMS}

Several barriers exist to establishing a stroke systems care program, as detailed in Table 2. The support and involvement of the hospital administration is essential to success, as is multidisciplinary agreement that such a program will benefit patients.

Other potential points of resistance revolve around the financial impact of implementing a stroke systems approach to care. The proposed stroke systems care plan is consistent with meeting nationally recognized quality improvement standards; however, the current health care market forces demand accountability for health care expenditures. Increasingly, payers are turning to the Joint Commission on Accreditation of Healthcare Organizations (JCAHO) and the National Committee for Quality Assurance (NCQA) evaluations to determine quality of care at various institutions. These programs encourage the use of standardized treatment protocols consistent with the concept of systems approach to care. Moreover, stroke care is a JCAHO quality measure and 
thus may have a financial impact on hospitals. It is possible that implementing standardized procedures for stroke care may reduce the cost of care. Information about the JCAHO Disease Specific Certification for Acute Stroke Care can be accessed at http://www.jointcommission.org/. ${ }^{7}$

Once there is agreement that a stroke system should be developed, a multidisciplinary team should be established. A multidisciplinary team may include hospitalists, neurologists, neurosurgeons, emergency medicine physicians, diagnostic and interventional radiologists, nurses, physiotherapists, occupational therapists, speech and language therapists, and social workers. However, the components of the multidisciplinary team may vary depending on the available staff and financial resources at different stroke centers. Assuring all participants in the system that their input is valued can improve communication among stroke specialists, hospitalists, and primary care clinicians. This team is responsible for evaluation of current procedures and development of algorithms, discharge forms, patient education, and preprinted orders.

The task of developing a cohesive plan for stroke care may appear onerous. Existing diagnostic and treatment procedures may be poorly designed or organized. However, multiple online sources provide tools for every aspect of stroke systems care. Information about evidence-based stroke care practices is available as part of the American Heart Association (AHA)/ASA "Get with the Guidelines-Stroke" program and can be accessed at http://www.strokeassociation.org. ${ }^{8}$ Standardized admission orders, patient education materials, and data-monitoring methods are available at this site. Hospitalist-specific information may also be found in the Stroke Resource Room of the Society of Hospital Medicine Web site (http://www.hospitalmedicine.org). ${ }^{9}$ A similar array of tools can be found at the PROTECT Web site (http://strokeprotect.mednet.ucla.edu). ${ }^{10}$ These materials are designed to facilitate integration of secondary prevention practices into stroke systems care.

A stroke system of care is a dynamic process. The multidisciplinary team may also be responsible for continuous monitoring and reporting of the efficiency and impact of the system and providing feedback to other staff and administration. Protocols should be revised regularly to account for new evidence-based treatments and to stream- line their use. The Canadian Stroke Systems Coalition recommends that a comprehensive and efficient system include prevention, prehospital and emergency care, hospital care, rehabilitation, reintegration into the community, surveillance, and research. ${ }^{11}$ Hospital staff should be educated in core competencies in hospital medicine as well as any changes to protocols made over time. Protocols that facilitate communication among health care providers should also be developed, and hospitalists may play a central role in this process. Accurate and timely transfer of patient information from the emergency department to the stroke center or ward is imperative.

\section{FOCUSING ON INPATIENT CARE}

Clinical pathways for inpatient care should be designed to limit stroke progression as much as possible. ${ }^{3}$ The Brain Attack Coalition (BAC) provides a resource for clinical pathways implemented at various institutes in the United States, including the Stanford Stroke Center, the Cleveland Clinic Foundation, and Thomas Jefferson University Hospital, among others (http://strokesite.org/pathways/pathways.html). ${ }^{12}$ Between $30 \%$ and $40 \%$ of acute stroke patients will show signs of stroke progression in the first week following stroke onset. Stroke can affect multiple systems, and symptom progression as a result of cerebral edema, hemorrhagic conversion, or additional strokes may require surgical intervention and warrants consultation with a neurologist. ${ }^{13}$

A neurologist should be available to the stroke system patients at all times, and ideally, all acute stroke patients should be evaluated by a neurologist specializing in the evaluation and treatment of patients with stroke. ${ }^{14}$ There are several stroke scales available to evaluate stroke patients, including the Barthel Index, the Glasgow outcome scale, the Modified Rankin Scale, the National Institutes of Health Stroke Scale, and the Hunt and Hess Classification of Subarachnoid Hemorrhage (http:// www.stroke-site.org/stroke_scales/stroke_scales. html). ${ }^{15}$ These scales aid in the assessment of severity of stroke and may be helpful for hospitalists and other health care providers to recognize when a neurology consult is needed. The team should agree on criteria that indicate a neurological emergency, such as stroke progression, and other reasons for immediate consultation with a neurologist, such as worsening of stroke, stroke in a 
young patient, or uncertain diagnosis. These criteria should be included as an established part of the clinical pathway.

Common complications of stroke, such as myocardial infarction, deep vein thrombosis, pulmonary embolism, urinary tract infections, aspiration pneumonia, dehydration, poor nutrition, skin breakdown, and metabolic disorders, should be anticipated, and preventive steps should be taken. The measures to prevent the above complications of stroke need to be initiated in the emergency department. $^{3}$

Management of existing comorbid conditions is another key part of subacute stroke care. Given that $85 \%$ of all hospitalists have a background in internal medicine, management of comorbid conditions such as diabetes and hypertension is an area in which hospitalists have professional competence. Patient history and use of prescription medications prior to stroke should be reviewed whenever possible and incorporated into shortterm and long-term treatment plans. Patients with diabetes in particular may benefit more from rigorous control of blood pressure and lipids compared with other patients. ${ }^{16}$

Secondary stroke prevention should start as early as considered safe. Diagnosis of stroke subtype, often accomplished in the emergency department, establishes suitability for antithrombotics and optimal management strategy. Patients who receive a diagnosis of stroke secondary to cardioembolic atrial fibrillation should be treated with an anticoagulant after the acute period. Aspirin can be used for those individuals unable to use anticoagulants. ${ }^{16}$ For those individuals with stroke of noncardioembolic origin, particularly those with atherosclerosis and lacunar or cryptogenic infarcts, antiplatelet agents are recommended. ${ }^{14}$

A multimodal prevention strategy is recommended to manage blood pressure and dyslipidemia poststroke. An algorithm for managing blood pressure soon after stroke has been developed by the PROTECT program (Fig. 2). ${ }^{10}$ Antihypertensives, usually a combination of an ACE inhibitor and a thiazide diuretic, can be initiated at low doses 4872 hours after stroke. A longer delay is recommended for patients with large infarcts or evidence of uncontrolled hypertension. ARBs may be substituted for ACE inhibitors. ${ }^{10}$ Target blood pressures should be determined using the Seventh Report of the Joint National Committee on Prevention, Detection, Evaluation, and Treatment of High

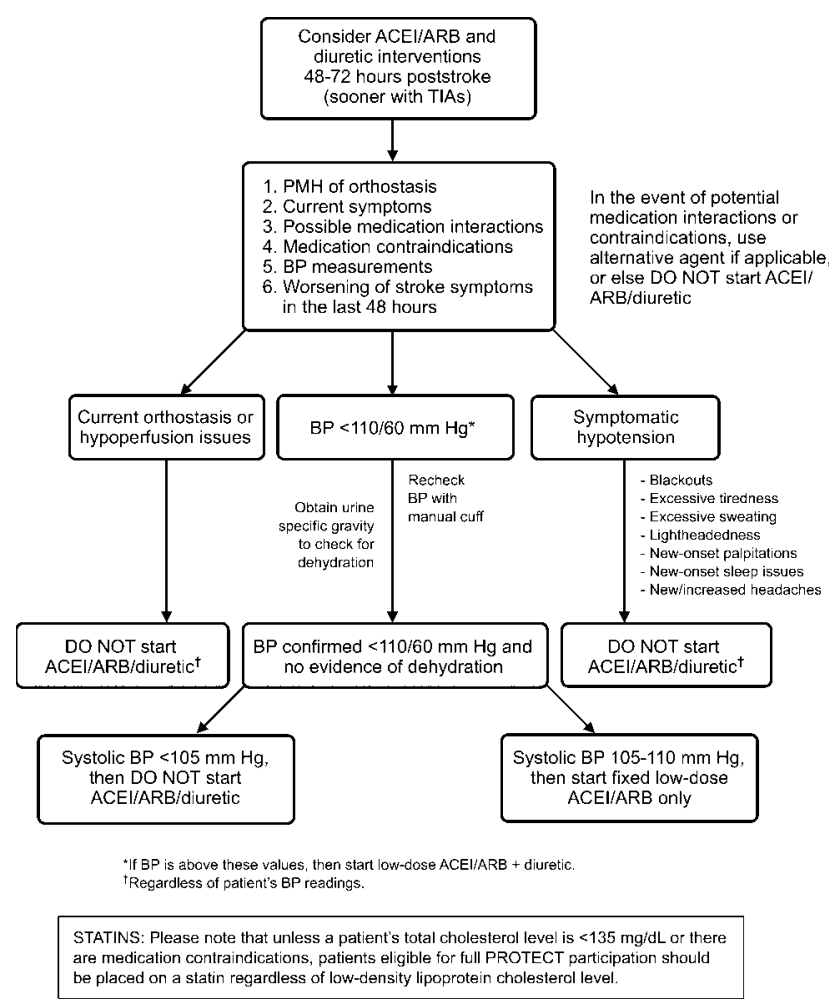

FIGURE 2. PROTECT antihypertensive treatment algorithm. Adapted with permission from UCLA Stroke PROTECT Program. Available at http://strokeprotect.mednet.ucla.edu; accessed September 12, $2007^{10}$ (ACEl, angiotensinconverting enzyme inhibitor; $\mathrm{ARB}$, angiotensin receptor blocker; $\mathrm{BP}$, blood pressure; LDL, low-density lipoprotein; PMH, past medical history; TIA, transient ischemic attack).

Blood Pressure. ${ }^{17}$ In general, even a reduction of $10 / 5 \mathrm{~mm} \mathrm{Hg}$ has been shown to be beneficial. ${ }^{16}$

Statins are recommended for all patients with elevated serum lipids unless treatment with statins is contraindicated. The recommended target level for low-density lipoprotein cholesterol is below $100 \mathrm{mg}$ / $\mathrm{dL}$ for individuals with coronary heart disease and symptomatic atherosclerosis. A target below $70 \mathrm{mg} /$ $\mathrm{dL}$ may be appropriate for patients at very high risk. ${ }^{16}$

Prior to discharge, patients or their caregivers should be given prescriptions adequate to cover the time until postdischarge follow-up visit. The responsible persons need to be made aware that some medications such as antihypertensives will require dosage adjustments by an outpatient physician, and the timing of the follow-up visit may need to be arranged accordingly.

The importance of stroke risk reduction should be part of predischarge patient education, 
along with a list of the warning signs of stroke. Adherence to the treatment regimen, including lifestyle changes and medications, should be emphasized. Patients or their caregivers should be educated about identifying adverse events and a plan to address them. Understanding that some adverse effects (eg, headache with aspirin plus extended-release dipyridamole) are likely to be transient may prevent unnecessary discontinuation of treatment and reduce anxiety.

Patient and caregiver education can be reinforced by providing standardized patient education materials that can be found in the Stroke Resource Room at the Society of Hospital Medicine Web site (http://www.hospitalmedicine.org), ${ }^{9}$ PROTECT (http://strokeprotect.mednet.ucla.edu), ${ }^{10}$ ASA (http://www.strokeassociation.org), ${ }^{8}$ and AHA (http://www.americanheart.org) ${ }^{18}$ Web sites.

Transfer of patient information to outpatient health care providers is a critical step in stroke systems care. Notes indicating any need for medication dose adjustment must be included. Discharge summaries should be available to primary care providers, neurologists, and rehabilitation specialists prior to follow-up visits. The use of electronic forms that can be faxed or sent by Email can shorten delivery time considerably. In lieu of electronic delivery, physician letters can be used, and prototypes are available at the resource Web sites. Whenever possible, a follow-up phone call to the primary care physician provides the best means to ensure clear communication.

\section{SUMMARY}

Hospitalists are well qualified to lead quality focused patient care initiatives at their institutions. Use of standardized protocols to reduce the risk of secondary stroke is proven to increase appropriate prescribing at discharge, which in turn improves patient adherence to evidencebased therapy. Multidisciplinary communication, including communication with outpatient clinicians, facilitates the transition from inpatient to outpatient health care providers.

In addition to improving patient care, use of standardized protocols is tracked by JCAHO and offers assurance to payers that a particular hospital and its staff are committed to quality care. Establishing protocols is made relatively easy by the online availability of materials that can be adapted to various hospital settings.
Address for correspondence and reprint requests: Alpesh Amin, MD, MBA UC Irvine School of Medicine, 101 The City Drive South, Building 26, ZC4076H, Orange, CA 92868. Fax (714) 456-7182; E-mail: anamin@uci.edu

Received 2 November 2007; revision received 26 March 2008; accepted 28 March 2008.

\section{REFERENCES}

1. Reeves MJ, for the Paul Coverdell Prototype Registries Writing Group. Acute stroke care in the US: results from 4 pilot prototypes of the Paul Coverdell National Acute Stroke Registry. Stroke. 2005;3:1232-1240.

2. Committee on Quality of Health Care in America, Institute of Medicine. Crossing the Quality Chasm: A New Health System for the $21^{\text {st }}$ Century. Washington, DC: National Academies Press; 2001.

3. Schwamm LH, Pancioli A, Acker JE 3rd, et al. American Stroke Association's Task Force on the Development of Stroke Systems. Recommendations for the establishment of stroke systems of care: recommendations from the American Stroke Association's Task Force on the Development of Stroke Systems. Circulation. 2005;111:1078-1091.

4. Ovbiagele B, Saver JL, Fredieu A, et al. PROTECT: a coordinated stroke treatment program to prevent recurrent thromboembolic events. Neurology. 2004;63:1217-1222.

5. Ovbiagele B, Saver JL, Fredieu A, et al. In-hospital initiation of secondary stroke prevention therapies yields high rates of adherence at follow-up. Stroke. 2004;35:2879-2883.

6. Fonarow GC, Gawlinski A, Moughrabi S, Tillisch JH. Improved treatment of coronary heart disease by implementation of a cardiac hospitalization atherosclerosis management program (CHAMP). Am J Cardiol. 2001;87:819-822.

7. Joint Commission on Accreditation of Hospital Organizations web site. Available from URL: http://www. jointcommission.org/. Accessed September 12, 2007.

8. American Stroke Association. Get with the Guidelines. Available at: www.strokeassociation.org/presenter.jhtml? identifier $=1200037$. Accessed September 12, 2007.

9. Society for Hospital Medicine. Stroke Research Room. Available at: http://www.hospitalmedicine.org/AM/Template. cfm?Section=Quality_Improvement_Resource_Rooms\&Template $=/ \mathrm{CM} / \mathrm{HTMLDisplay.cfm \& ContentID}=6566$. Accessed September 12, 2007.

10. UCLA Stroke PROTECT Program. Available at: http://strokeprotect.mednet.ucla.edu. Accessed September 12, 2007.

11. Wilson E, Taylor G, Phillips S, Stewart PJ, et al. Canadian Stroke Systems Coalition. Creating a Canadian stroke system. CMAJ. 2001;164:1853-1855.

12. Brain Attack Coalition. Pathways. Available at: http:// stroke-site.org/pathways/pathways.html. Accessed January $28,2008$.

13. Dávalos A, Toni D, Iweins F, Lesaffre E, Bastianello S, Castillo J. Neurological deterioration in acute ischemic stroke: potential predictors and associated factors in the European Cooperative Acute Stroke Study (ECASS) I. Stroke. 1999;30: 2631-2636.

14. Alberts MJ, Latchaw RE, Selman WR, et al. Recommendations for comprehensive stroke centers: a consensus statement from the Brain Attack Coalition. Stroke. 2005;36: $1597-1618$. 
15. Brain Attack Coalition. Stroke scales. Available at: http:// www.stroke-site.org/stroke_scales/stroke_scales.html. Accessed January 28, 2008.

16. Sacco RL, Adams R, Albers G, et al. American Heart Association; American Stroke Association Council on Stroke; Council on Cardiovascular Radiology and Intervention; American Academy of Neurology. Guidelines for prevention of stroke in patients with ischemic stroke or transient ischemic attack: a statement for healthcare professionals from the American Heart Association/American Stroke Association Council on Stroke. Stroke. 2006;37:577-617.

17. Chobanian AV, Bakris GL, Black HR, et al. Seventh Report of the Joint National Committee on Prevention, Detection, Evaluation, and Treatment of High Blood Pressure. JAMA. 2003;42:1206-1252.

18. American Heart Association. Available at: http://www. americanheart.org/ presenter.jhtml?identifier $=1200000$. Accessed September 12, 2007. 